\title{
Produção acadêmica da enfermagem acerca dos temas HIV e aids: um estudo histórico-social
}

\author{
Nursing academic production on the topics of HIV and aids: a historical-social study \\ Producción académica de enfermería sobre los temas VIH y sida: un estudio histórico-social
}

\section{Stéfany Petry', Maria Itayra Padilha ${ }^{\mathrm{II}}$, Ana Rosete Maia ${ }^{\mathrm{III}}$, Gabriela Beims Gapski ${ }^{\mathrm{IV}}$}

Resumo: Objetivo: conhecer os trabalhos de conclusão de curso sobre a temática HIV/aids no Curso de Graduação em Enfermagem da Universidade Federal de Santa Catarina no período de 1990 a 2017. Método: pesquisa qualitativa, histórico-social, de fontes documentais acerca da temática HIV/aids no Curso de Graduação em Enfermagem da UFSC. Resultados: foram levantados 773 trabalhos de conclusão do cursos, sendo que 25 (3,23\%) se enquadravam na temática. Identificaram-se estudos voltados a assistência de pacientes com HIV, promoção à saúde de adolescentes, e os riscos dos profissionais da área da saúde. Considerações finais: observouse que o tema ainda é pouco investigado nos trabalhos de conclusão de curso, apesar da ampliação nos últimos três anos, faz-se necessário a realização de pesquisas que contribuam para redução do estigma social da doença e também que possam auxiliar na promoção e prevenção da saúde da população.

Descritores: Estudantes de enfermagem; Pesquisa em enfermagem; Síndrome de Imunodeficiência Adquirida; Monografia

Abstract: Aim: to know the final graduation paper productions on HIV/AIDS in the Nursing Undergraduate Course of the Federal University of Santa Catarina from 1990 to 2017. Method: qualitative research, socialhistorical, of documented sources on the subject of HIV/AIDS in the Undergraduate Nursing Course of UFSC. Results: Of the 773 final graduation papers found, 25 (3.23\%) were in the subject area. Studies aimed at the care of HIV patients, adolescent health promotion, and the risks of health professionals were identified. Final considerations: it was observed that the subject is still little investigated in final graduation papers, despite the

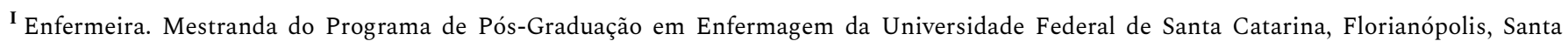
Catarina, Brasil. E-mail: stefanypetry@hotmail.com. Orcid: https://orcid.org/0000-0001-9713-247X

II Enfermeira. Professora Doutora do Programa de Pós-Graduação em Enfermagem da Universidade Federal de Santa Catarina, Florianópolis, Santa Catarina, Brasil. E-mail: itayra.padilha@ufsc.br. Orcid: https://orcid.org/0000-0001-9695-640X

III Enfermeira. Professora Doutora do Departamento de Enfermagem da Universidade Federal de Santa Catarina, Florianópolis, Santa Catarina, Brasil. E-mail: anamaia8@hotmail.com. Orcid: https://orcid.org/0000-0002-3353-1225

IV Estudante de Graduação em Enfermagem pela Universidade Federal de Santa Catarina, Florianópolis, Santa Catarina, Brasil. E-mail: gabriela_beims@hotmail.com. Orcid: https://orcid.org/0000-0002-5627-8824
} 
increase in the last three years. It is necessary to carry out researches that contribute to the reduction of the disease's social stigma and that can help in promoting and preventing the health of the population.

Descriptors: Students, Nursing; Nursing Research; Acquired Immunodeficiency Syndrome; Monograph

Resumen: Objetivo: conocer los trabajos finales de curso sobre VIH/SIDA, en el Curso de Enfermería, de la Universidad Federal de Santa Catarina (UFSC), entre 1990 y 2017. Método: investigación cualitativa, sociohistórica, a partir de fuentes documentales, específicamente, las monografías sobre el tema VIH/SIDA en el curso de enfermería de la UFSC. Resultados: fueron encontrados 773 trabajos finales de curso, 25 (3,23\%) realizados sobre esa área temática. Identificamos estudios direccionados a la atención de los pacientes con VIH, a la promoción de la salud de los adolescentes y a los riesgos de los profesionales de la salud. Consideraciones finales: el tema aun es poco investigado en trabajos finales de curso, aunque el número de investigaciones aumentó en los últimos tres años, es necesario desarrollar más trabajos que contribuyan para la

Descriptores: Estudiantes de Enfermería; Investigación en Enfermería; Síndrome de Inmunodeficiencia Adquirida; Monografía

\section{Introdução}

O curso de Graduação em Enfermagem tem como perfil a formação do enfermeiro generalista, humanista, crítico e reflexivo. Esse profissional torna-se qualificado para o exercício de enfermagem quando possui uma base científica e intelectual pautada em princípios éticos, por isso, o programa pedagógico do curso deve oferecer atividades complementares sendo por meio de estudo e práticas independentes presenciais e/ou à distância; monitorias e estágios; programas de iniciação científica; programas de extensão; estudos complementares e cursos realizados em outras áreas. ${ }^{1}$

Quando trata-se de pesquisa, a inserção precoce do aluno deve ser estimulada, porém pode ser realizada em vários momentos, levando em conta a estrutura curricular oferecida pelo curso. Assegurando a articulação entre o ensino, pesquisa e extensão/assistência, e também estimule a realização de experimentos e/ou projetos de pesquisa para uma evolução dos modelos explicativos do processo saúde-doença. Haja visto que tanto durante a formação como na conclusão de curso as pesquisas são essenciais. ${ }^{1}$ 
Nos dias atuais a pesquisa e a tecnologia ocupam um lugar de destaque nas universidades brasileiras. Os programas de pós-graduação aliados com ações de incentivo e recursos destinados à pesquisa contribuem para o aumento das produções científicas dentro das universidades e facilitam o acesso dos alunos da graduação no meio científico. ${ }^{2}$

Tendo em vista a importância da pesquisa na graduação em Enfermagem, aliado as Diretrizes Curriculares Nacionais (DCN), vem sendo discutido nos últimos anos mudanças para reforçar a integração entre a teoria e a prática, assim como a formação interdisciplinar, participação dos enfermeiros dos serviços nas atividades práticas e estágios curriculares supervisionados, e o estabelecimento de áreas prioritárias de formação que visem atender o quadro epidemiológico da população e os princípios do Sistema Único de Saúde. ${ }^{3}$

Em concordância a esses pontos, é durante a graduação que os estudantes de enfermagem desenvolvem o Trabalho de Conclusão de Curso (TCC), com auxílio e orientação de um docente. Esses estudos denominados monografias e posteriormente TCC tem como objetivo conhecer as tendências temáticas dos acadêmicos de Enfermagem. Verificou que os tipos de pesquisa mais utilizadas pelos acadêmicos de Enfermagem são: pesquisa de campo, pesquisa bibliográfica ou de revisão teórica, pesquisa documental e pesquisa histórica. ${ }^{4} \mathrm{O}$ aprendizado do aluno com relação à pesquisa também ocorre por meio da inserção do estudante em grupos de pesquisa, proporcionando convívio e aprendizado com outros estudantes de graduação e de pósgraduação em nível mestrado e doutorado. A atividade extracurricular também faz parte do processo de formação dos enfermeiros, sendo um mecanismo auxiliar na proposta pedagógica do curso, da Instituição de Ensino e na formação da competência científica. ${ }^{5}$

Faz-se importante a inserção do estudante de enfermagem nos grupos de pesquisa, ao considerar que os estudos realizados pelos grupos, orientados por docentes pesquisadores promovem a aproximação do ato de investigar, trazendo a reflexão crítica acerca de possíveis soluções para problemas da prática assistencial, gerencial e de ensino da profissão. Essas 
Produção acadêmica da enfermagem acerca dos temas hiv e aids: um estudo histórico-social | 4

questões irão promover aos futuros profissionais de enfermagem oportunidades de pesquisa para a sua prática tanto no que se refere à busca de respostas para um problema de saúde, quanto a habilidade de escolha de estudos com melhor nível de evidência. ${ }^{6}$ Esse processo se faz necessário através da compreensão do ensino da pesquisa na graduação, com a intenção de formar enfermeiros cada vez mais qualificados e fortalecer a profissão como ciência em construção. ${ }^{7}$

Na Universidade Federal de Santa Catarina (UFSC), segundo o currículo instituído no ano de 2011, o curso passou de oito para dez semestres, e nas duas últimas fases do curso o aluno desenvolve o seu trabalho de conclusão de curso, que pode ocorrer de duas maneiras: pesquisa seguindo os trâmites de aprovação do Comitê de Ética em Pesquisa ou diagnóstico, resolução e avaliação fundamentada com a intenção de buscar soluções aos problemas da prática assistencial, educacional e gerencial. ${ }^{8}$

Um dos temas discutidos nos TCC é relativo ao HIV/aids, o qual foi identificado em 1982 e desde a sua descoberta carrega um estigma social. É importante o reconhecimento da doença como um problema de saúde pública e que a comunidade científica auxilie na propagação de informações a fim de contribuir na construção de medidas preventivas. Para que essas concepções mudem, é indispensável a realização de pesquisas que evidenciem o real significado da doença, tendo as instituições de ensino papel importante na atribuição de significados e valorização dos aspectos referentes à temática e sua apropriação pelos futuros profissionais enfermeiros. ${ }^{9}$

Tendo em vista que a o HIV/aids ainda continua sendo um grave problema de saúde no Brasil e no mundo, a enfermagem tem grande participação nas ações de promoção de saúde, prevenção e prática assistencial. ${ }^{10}$ Vale apontar, que o HIV/aids é considerado um problema de saúde pública, pois o mesmo apresenta impacto no indivíduo em termos de anos de vida perdidos, a extensão da incapacidade, aspectos financeiros ligados ao tratamento, dor e 
desconforto e impacto na família. Assim como traz, também, impactos na sociedade ligados a mortalidade, morbidade e custos financeiros com relação ao tratamento. Outro aspecto relevante como critério para elencar a doença enquanto problema de saúde pública está associado ao seu potencial epidêmico. ${ }^{11-12}$

Diante do exposto, este estudo tem por objetivo conhecer os trabalhos de conclusão de curso sobre a temática HIV/aids no Curso de Graduação em Enfermagem da UFSC no período de 1990 a 2017.

\section{Método}

Trata-se de uma pesquisa exploratória descritiva de abordagem qualitativa históricosocial do tipo documental. A utilização de documentos na pesquisa proporciona o alcance de informações objetivas no contexto subjetivo da história. Os documentos aparecem como fontes e podem ser materiais contemporâneos ou retrospectivos que contêm matérias orais e escritas, textos, livros e outros materiais bibliográficos. A pesquisa histórica tem a documentação como método e apresenta processos de escolha, organização, análise e síntese. As técnicas da pesquisa documental correspondem ao conjunto de operações de coleta, observação e tratamento das informações, para converter os fatos em dados analisáveis. ${ }^{13}$

A apresentação de um TCC na graduação em Enfermagem da UFSC passou a ser requisito em 1978, porém só foram obtidos dados das produções a partir de 1982 em um catálogo produzido pelo Departamento de Enfermagem da UFSC na qual consta os TCC de 1982 a $1997 .{ }^{14}$ Os dados correspondentes de 1998 até 2017 foram obtidos pelas produções disponíveis na Biblioteca Universitária da UFSC e no seu no acervo digital (Pergamum), na Biblioteca Universitária do Hospital Universitário Professor Polydoro Ernani de São Thiago e nos arquivos do Departamento de Enfermagem da UFSC. Além da cooperação e divulgação dos trabalhos por 
professores responsáveis pelas disciplinas vinculadas à elaboração dos trabalhos de conclusão de curso. A partir desse levantamento foi possível encontrar os TCC relativos ao tema HIV/aids.

É importante destacar que em 2011 ocorreu a mudança do currículo do curso, na qual modificou-se o tempo de duração de oito para dez semestres, o que ocasionou um hiato de tempo quanto às turmas se formando. Dessa maneira, não existem dados de TCC referentes ao primeiro semestre de 2015.

A coleta e organização dos dados ocorreram no período de maio a agosto de 2018. Foram levantados 773 TCCs, todos foram inseridos em uma planilha no Microsoft Excel e classificados por título, autor, orientador, referencial teórico e ano. Para uma análise inicial foram lidos todos os títulos e resumos dos trabalhos, após essa etapa, separou-se aqueles que abordavam a referente temática em seus títulos e resumos utilizando explicitamente o termo "HIV” ou "aids”, ou ainda, trabalhos que continham assuntos como: sexualidade, saúde reprodutiva, violência sexual e acidentes biológicos. Posteriormente foi realizada nova leitura dos títulos e resumos dos resultados selecionados, e para classificação e compreensão foi feita leitura da metodologia para chegar ao resultado final do quantitativo de TCCs a serem analisados.

Após a seleção dos estudos, foi realizado detalhamento dos dados acerca de cada pesquisa no que diz respeito ao ano de publicação, quais os docentes orientadores e de que maneira a temática HIV ou aids estavam sendo abordadas em seus diversos campos e cenários de pesquisa. Por se tratar de uma pesquisa documental com a utilização de dados de acesso público não se faz necessária a aprovação de Comitê de Ética em Pesquisa com Seres Humanos.

\section{Resultados}

Foram produzidos 773 TCCs pelos estudantes do Curso de Graduação em Enfermagem da UFSC entre os anos de 1982 a 2017. Percebe-se que mesmo sendo um período de 35 anos de produções houve apenas 25 estudos na área de HIV/aids, o que representa 3,23\% da produção 
total. Além disso, o primeiro estudo sobre a temática foi realizado no ano de 1990, após 8 anos de identificação da doença.

Nem todos os estudos encontrados estavam enquadrados ou estudavam especificamente o HIV/aids, porém de alguma forma estavam intrinsecamente associados a temática.

Quadro 1 - Trabalhos de conclusão de curso de graduação em Enfermagem da UFSC com a temática HIV/aids de 1990 até 2017, Florianópolis, 2018.

\begin{tabular}{|c|c|c|}
\hline Ordem & Título & Ano \\
\hline 1 & Assistência de enfermagem ao portador do HIV e doentes de aids & 1990 \\
\hline 2 & $\begin{array}{l}\text { Aplicação do processo de enfermagem na assistência de clientes do } \\
\text { serviço de atendimento as necessidades psicossociais da UFSC (SANPS) }\end{array}$ & 1993 \\
\hline 3 & $\begin{array}{l}\text { Prevenção de doenças sexualmente transmissiveis com adolescentes em } \\
\text { colégio estadual de Florianópolis }\end{array}$ & 1993 \\
\hline 4 & $\begin{array}{l}\text { Cuidado de Enfermagem aos portadores do HIV/aids no hospital e } \\
\text { domicílio - uma nova proposta assistencial }\end{array}$ & 1994 \\
\hline 5 & $\begin{array}{l}\text { A enfermagem cuidando da mulher/mãe com HIV ou aids através da } \\
\text { construção da relação de ajuda }\end{array}$ & 1994 \\
\hline 6 & $\begin{array}{l}\text { Cuidado de Enfermagem ao portador de HIV/aids com base na teoria das } \\
\text { Necessidades Humanas Básicas }\end{array}$ & 1996 \\
\hline 7 & $\begin{array}{l}\text { Assistência de Enfermagem ao Portador do HIV/aids utilizando o marco } \\
\text { conceitual dos estressores e enfrentamentos }\end{array}$ & 1996 \\
\hline 8 & $\begin{array}{l}\text { A Enfermagem interagindo com a família com HIV/aids em um hospital } \\
\text { pediátrico, em busca do alcance dos objetivos }\end{array}$ & 1996 \\
\hline 9 & $\begin{array}{l}\text { Assistência de Enfermagem aos (com) viventes de HIV/aids utilizando o } \\
\text { referencial teórico de Leininger }\end{array}$ & 1997 \\
\hline 10 & $\begin{array}{l}\text { Estudo prospectivo sobre métodos contraceptivos entre as pacientes do } \\
\text { ambulatório de ginecologia do Hospital Universitário conhecimento e } \\
\text { uso }\end{array}$ & 1998 \\
\hline 11 & $\begin{array}{l}\text { Compartilhando, através do Cuidado em Enfermagem, o saber em } \\
\text { planejamento familiar, buscando a promoção do autocuidado em saúde, } \\
\text { aos indivíduos em idade fértil }\end{array}$ & 1999 \\
\hline
\end{tabular}


Produção acadêmica da enfermagem acerca dos temas hiv e aids: um estudo histórico-social I 8

\begin{tabular}{|c|c|c|}
\hline 12 & $\begin{array}{l}\text { Assistência de enfermagem ao portador de HIV/aids com base na teoria } \\
\text { das necessidades básicas }\end{array}$ & 2000 \\
\hline 13 & Assistência à saúde do adolescente: Direitos Reprodutivos & 2003 \\
\hline 14 & $\begin{array}{l}\text { Violência Sexual: a enfermagem promovendo o autocuidado através da } \\
\text { educação em saúde a adolescente vitimizado }\end{array}$ & 2003 \\
\hline 15 & $\begin{array}{l}\text { Promovendo a autonomia para o cuidado humanizado ao portador de } \\
\text { HIV/aids em tratamento por anti-retroviral fundamentado na teoria de } \\
\text { Jean Watson }\end{array}$ & 2004 \\
\hline 16 & $\begin{array}{l}\text { A prática de enfermagem como processo educativo em saúde: a } \\
\text { contribuição dos agentes comunitários na prevenção da gravidez precoce } \\
\text { e doença sexualmente transmissíveis }\end{array}$ & 2005 \\
\hline 17 & A história de vida e as percepções do paciente ao viver com aids & 2010 \\
\hline 18 & $\begin{array}{l}\text { Perfil dos acidentes de trabalho entre profissionais de enfermagem com } \\
\text { exposição a material biológico no período de } 2005 \text { a 2011: realidade de um } \\
\text { Hospital do sul do Brasil }\end{array}$ & 2012 \\
\hline 19 & $\begin{array}{l}\text { Gerenciando o cuidado de enfermagem ao adolescente que vive com } \\
\text { HIV/aids por transmissão vertical }\end{array}$ & 2012 \\
\hline 20 & Repercussão do acidente de trabalho na vida dos profissionais & 2013 \\
\hline 21 & $\begin{array}{l}\text { A mulher que vive com HIV/aids e a (im)possibilidade de amamentação: } \\
\text { uma revisão integrativa }\end{array}$ & 2014 \\
\hline 22 & $\begin{array}{l}\text { Intenções comportamentais apresentadas pelos homens diante dos } \\
\text { resultados sorológicos de doenças sexualmente transmissíveis }\end{array}$ & 2014 \\
\hline 23 & $\begin{array}{l}\text { Compreendendo o cuidado domiciliar prestado por mães HIV's aos } \\
\text { lactantes expostos ao HIV }\end{array}$ & 2016 \\
\hline 24 & $\begin{array}{l}\text { Conhecimentos e atitudes de autocuidado de estudantes de enfermagem } \\
\text { diante das infecções sexualmente transmissíveis }\end{array}$ & 2017 \\
\hline 25 & $\begin{array}{l}\text { Promoção da saúde sexual e reprodutiva: uma conversa com adolescentes } \\
\text { sobre direitos sexuais e reprodutivos }\end{array}$ & 2017 \\
\hline
\end{tabular}

Fonte: Próprias autoras.

Classificaram-se os TCCs sobre a temática HIV/aids em diversas áreas de estudo: 09 produções referente a saúde do adulto (36\%), 05 saúde do adolescente (20\%), 04 produções na saúde da mulher (16\%), 02 saúde do trabalhador (8\%), 02 em saúde pública (8\%), 01 com foco na gerência (4\%), 01 em saúde da criança (4\%), e 01 com foco na saúde dos estudantes de 
enfermagem (4\%). Os cenários dos estudos foram: 12 estudos ocorreram no hospital (48\%), 05 em escolas (20\%), 04 na Atenção Primária em Saúde (16\%), 02 no domicílio (8\%), 01 Centro de Testagem e Aconselhamento (CTA) (4\%) e 01 na universidade (4\%).

Quando associado essas produções a docentes orientadores, observou-se que tiveram 10 docentes da universidade preocupados em desenvolver pesquisas com a temática durante esses 35 anos. Se destacando com orientação de 06 trabalhos a Prof ${ }^{\mathbf{a}}$ Dr $^{\mathbf{a}}$ Maria Helena Bittencourt Westrupp, seguida pela Prof ${ }^{a}$ Dr ${ }^{a}$ Maria Itayra Padilha com 04 orientações.

\section{Discussão}

Pesquisar sobre algum tema envolve a busca do conhecimento mais recente sobre determinado assunto e a criação de questões a serem respondidas a fim de construir um projeto. As tarefas necessárias para desenvolver um projeto, são planejadas e executadas de acordo com o método adequado, culminando com à discussão dos resultados e a elaboração das conclusões do estudo. ${ }^{15}$

Desde a identificação do HIV/aids em 1982, os estudos científicos foram se intensificando a fim de conhecer melhor a doença e desenvolver uma terapêutica. Historicamente, o primeiro caso de aids notificado no Estado de Santa Catarina foi em agosto de 1984. Porém, o primeiro trabalho de conclusão de curso produzido na Graduação em Enfermagem da UFSC em 1990, esse estudo pioneiro buscou informar o que é esse vírus, como ocorre a transmissão, quais os sinais e sintomas, como o organismo reage diante desse vírus, tendo como objetivo geral a assistência de enfermagem ao portador de HIV/aids. ${ }^{16}$

O surgimento da epidemia da aids no Brasil ocorre em 1982 quase que simultaneamente ao cenário internacional, as autoridades sanitárias detectam a possibilidade de transmissão da aids através do ato sexual, uso de drogas injetáveis e exposição a sangue e derivados. A doença conhecida até então com o nome provisório de Doença dos 5H: homossexuais, hemofílicos, 
haitianos, heroinômanos (usuários de heroína injetável) e prostitutas (hookers, em inglês) passa a ser chamada com nome definitivo de Síndrome da Imunodeficiência Adquirida (Sida, em espanhol, ou Aids, na sigla em inglês). ${ }^{17}$

É importante ressaltar que a transmissão do HIV entre os Usuários de Drogas Injetáveis (UDI) é bastante acentuada nas regiões Sul e Sudeste do Brasil. A infraestrutura econômica maior da região Sudeste possibilita o contato entre pessoas e serve de suporte para o tráfico de entorpecentes. A infecção pelo HIV entre UDI no estado de São Paulo está associado ao baixo custo da droga, o que acaba facilitando a sua disseminação entre os municípios e também atraindo ainda mais o usuário. ${ }^{18}$

Considerando o perfil epidemiológico da região de Santa Catarina, o Estado assume a segunda maior taxa de detecção de aids no país, de acordo com o Ministério da Saúde, com 31,9/100.000 habitantes. Em primeiro lugar, está o Rio Grande do Sul que apresenta taxa de 34,7/100.000 habitantes. A nível nacional, a taxa de detecção da aids é de 19,1/100.000 habitantes. No Estado de Santa Catarina, 12 municípios participam de um acordo firmado entre o Governo Estadual e o Governo Federal para priorizar ações de prevenção e maior abrangência do acesso ao diagnóstico de HIV/aids, por apresentarem as mais altas taxas de detecção no estado. São eles: Balneário Camboriú $(63,2)$, Itajaí $(61,9)$, São José $(57,7)$, Florianópolis $(53,7)$, Palhoça $(49,4)$, Lages $(44,1)$, Criciúma $(39,6)$, Blumenau $(39,5)$, Brusque $(35,0)$, Joinville $(30,6)$, Jaraguá do Sul $(29,3)$ e Chapecó $(29,2) .{ }^{19}$

Faz-se necessário assumir um olhar complexo sobre fatores como a epidemiologia, a prevenção e os programas de saúde em HIV/aids, contribuindo dessa maneira para uma constante evolução conceitual e científica que propicie um passo a frente no entendimento desses fatores e que traduza-se em práticas que desestabilizem o estigma, assim como a repercussão desse na gestão do cuidado. ${ }^{20}$ 
Com relação a temática pode-se ainda considerar os acidentes de trabalho dos profissionais de enfermagem por meio da exposição à materiais biológicos. A saúde do trabalhador e quais as condutas adotadas em um acidente de trabalho com material biológico, assim como a adesão dos profissionais diante das medidas de biossegurança fazem parte do cotidiano de trabalho da enfermagem. Os acidentes de trabalho podem ser compreendidos como doenças do trabalho, as quais são adquiridas pela função exercida pelo trabalhador no seu setor de trabalho, e os acidentes de percurso ao trabalho, caracterizados como decorrentes do trajeto entre residência e trabalho. ${ }^{21}$

Os profissionais de enfermagem ocupam áreas de operação/prestação de cuidados, como salas emergência, salas cirúrgicas e laboratórios, que os colocam em situações com risco maior de exposição, assim como os profissionais da limpeza que atuam na coleta e manipulação de material contendo materiais contaminados. ${ }^{22}$ No Brasil, os acidentes de trabalho com exposição a material biológico são de notificação compulsória e devem ser registrados no Sistema de Informações de Agravos de Notificações (SINAN). ${ }^{23}$ Os serviços de saúde possuem ambientes complexos, que apresentam riscos aos profissionais de saúde, os acidentes perfurocortantes continuam sendo um problema em relação a contaminação por material biologicamente contaminado, pois acarretam na possibilidade de transmissão de algum agente patógeno pelo sangue, como nos casos das hepatites B e C e do HIV. ${ }^{24}$

Outra área em que os TCCs tiveram ênfase foi na saúde da mulher/mãe com relação ao HIV/aids por transmissão vertical. A transmissão do HIV de mãe para filho pode ocorrer no útero, no período periparto ou por meio da amamentação. ${ }^{25}$ É fundamental o acesso das gestantes com resultado HIV positivo para aconselhamento, a fim de iniciar a prevenção da transmissão vertical do HIV. Sem o aconselhamento e conhecimento do status sorológico fica comprometido o processo de cuidado à mulher. 
Produção acadêmica da enfermagem acerca dos temas hiv e aids: um estudo histórico-social | 12

A prática do aconselhamento é complexa e traz diversos desafios na escuta e interação com os pacientes. A comunicação ao paciente do resultado sorológico para o HIV, por exemplo, é um momento delicado, especialmente quando o exame é realizado no momento do parto. ${ }^{26} \mathrm{O}$ diagnóstico deve ser dado o mais precoce possível, no pré-natal, uma vez comprovado as gestantes podem levar algum tempo para aceitar a situação e dar início aos procedimentos profiláticos para evitar a transmissão vertical do HIV. Nesse momento, a gestante se confronta com a possibilidade de que a doença se manifeste nela, ou em seu filho, caso as medidas de profilaxia sejam ineficientes. ${ }^{26}$

Alguns TCCs abordaram a temática da sexualidade, saúde reprodutiva com o público adolescente. Esses comportamentos sexuais e os relacionamentos sexuais são uma parte importante e necessária do desenvolvimento humano. É na adolescência que ocorre uma progressiva conquista da autonomia, elaboração de projetos, afirmação pessoal e social, e a procura da independência para chegar a vida adulta. Nessa fase, evidenciam-se os comportamentos socioafetivos e sexuais.

Os adolescentes estão cada vez mais precocemente iniciando sua vida sexual, antes mesmo de completarem 15 anos de idade e sem qualquer informação educativa que possa instruir sobre as questões da sexualidade, transformações do corpo, questões sobre a susceptibilidade às Infecções Sexualmente Transmissíveis (IST) e, principalmente, o HIV. Nessa idade, a consciência ingênua da realidade e o sentimento de onipotência somados a falta de informação, acarretam na atividade sexual sem proteção, o que os expõe aos diversos riscos. ${ }^{27}$

As práticas educativas com relação à educação sexual dos adolescentes faz-se importante nas ações dentro da escola. Nesse sentido, o enfermeiro, no desenvolvimento do Programa Saúde na Escola, deve assumir o papel de educador a fim de orientar e informar os adolescentes sobre a sexualidade e a prática do sexo seguro, livre de doenças e contaminação, esclarecendo dúvidas e curiosidades, e derrubando tabus. São vários os fatores que envolvem a questão da 
vulnerabilidade dos adolescentes as IST's, dentre elas: o início da vida sexual precoce, falta de informação sobre a realização do ato sexual, não utilização do preservativo, desigualdade de gênero, baixa renda e vulnerabilidade social. ${ }^{28}$

A temática possui grande abrangência devido à vasta gama de indivíduos que podem se encontrar em situações de vulnerabilidade à infecção. Percebe-se, que apesar de poucos TCCs com essa temática, os estudos permeiam o contexto histórico da doença, no qual havia uma preocupação inicial com o conhecimento de identificação, diagnóstico e tratamento da doença voltado para a população adulta que era o perfil da época, seguidos por estudos focados na contaminação de gestantes e o complexo processo de gestação e amamentação, e mais atual, estudos relacionados aos altos índices epidemiológicos na população jovem e a necessidade de educação e prevenção.

\section{Considerações finais}

O estudo demonstra a abrangência das pesquisas realizadas, que não estão somente focados nas pessoas com a doença, mas também na promoção da saúde em diferentes ambientes. O tema HIV/aids pode ser abordado de várias maneiras, estando especificamente ligados ao campo da saúde da mulher, a gestão, a saúde dos acadêmicos e profissionais de saúde, como também a usuários de drogas.

Dentre as monografias do curso de graduação em enfermagem ainda são poucos os estudos que abordam essa temática. É importante apontar que por ser um problema de saúde que atinge toda a população, de ambos os sexos, é necessário que as produções fossem mais numerosas a fim de promover ações de promoção e prevenção da doença, assim como ampliar debates que possam auxiliar no rebaixamento dos atuais índices epidemiológicos, principalmente no que se refere ao Estado de Santa Catarina. Considerou-se que por ser um tema envolto de estigmas, medos e vergonha, esses fatores possam influenciar na escolha do 
Produção acadêmica da enfermagem acerca dos temas hiv e aids: um estudo histórico-social | 14

tema do TCC, como também a maneira como a temática está sendo abordada durante a graduação.

É importante que o tema HIV/aids seja discutido durante a graduação de forma abrangente, não somente sua etiologia e prognóstico, mas como uma ação do profissional da saúde frente ao paciente. Entende-se que esse estudo pode influenciar tanto na escolha da temática como no possível objeto de estudo para a produção de outros trabalhos, pesquisas e artigos, como também alertar sobre o quanto esse campo precisa ser estudado e pesquisado a fim de aumentar e melhorar as informações sobre a doença, tendo em vista sua relevância acadêmica e social.

\section{Referências}

1. Brasil. Conselho Nacional De Educação. Resolução CNE/CES n. 3, de 7 de novembro de 2001. Institui Diretrizes Curriculares Nacionais do curso de graduação em enfermagem [Internet]. 2001 [acesso em 2018 jun 16]. Disponível em: http://portal.mec.gov.br/cne/arquivos/pdf/CES03.pdf

2. Zampieri VH, Santin JC, Sousa KMC, Lopes HSS, Sanches S, Silva HN, et al. Contribuições da iniciação científica no aprendizado e desenvolvimento dos estudantes no ensino superior. Rev Nativa [Internet]. 2018 [acesso em 2018 set 28];7(1):76-83. Disponível em: https://docplayer.com.br/135183205Contribuicoes-da-iniciacao-cientifica-no-aprendizado-e-desenvolvimento-dos-estudantes-no-ensinosuperior.html

3. Teixeira, E. Em tempos de novas Diretrizes Curriculares Nacionais (DCN) para o curso de graduação em enfermagem. Rev Enferm UFSM [Internet]. 2017 [acesso em 2018 set 28];7(2):3-4. Disponível em: https://periodicos.ufsm.br/reufsm/article/view/28859

4. Spindola T, Vileti JL, Henrique NN, Costa PS, Clos AC. A produção científica nas monografias de conclusão da graduação em enfermagem de uma instituição pública. Rev Enferm UERJ [Internet]. 2011 out-dez [acesso em 2018 jul 11];19(4):610-5. Disponível em: http://www.facenf.uerj.br/v19n4/v19n4a18.pdf

5. Moraes A, Guariente MHDM, Garanhani ML, Carvalho BG. The nurse training in research in the undergraduate education: teaching perceptions. Rev Bras Enferm [Internet]. 2018 [acesso em 2018 jul 11];71(Suppl 4):1556-63. Disponível em: http://www.scielo.br/scielo.php?script=sci_arttext\&pid=S0034$71672018001001556 \& \operatorname{lng}=\mathrm{pt}$ 
6. Azevedo IC, Silva RCL, Carvalho DPSRP, Cruz GKP, Lima JVH, Ferreira Júnior MAF. Importância do grupo de pesquisa na formação do estudante de enfermagem. Rev Enferm UFSM [Internet]. 2018 jun [acesso em 2018 jul 11];8(2):390-8. Disponível em: https://periodicos.ufsm.br/reufsm/article/view/26003

7. Piexak DR, Barlem JGT, Silveira RS, Fernandes GFM, Lunardi VL, Backes DS. A percepção de estudantes da primeira série de um curso de graduação em enfermagem acerca da pesquisa. Esc Anna Nery Rev Enferm [Internet]. 2013 [acesso em 2018 jul 11];17(1):68-72. Disponível em: http://www.scielo.br/pdf/ean/v17n1/10.pdf

8. Universidade Federal de Santa Catarina, Centro de Ciências da Saúde, Departamento de Enfermagem. O curso de Enfermagem [Internet]. 2012 [acesso em 2018 jun 16]. Disponível em: http://nfr.ufsc.br/files/2012/12/Programa-do-Curso-de-Enfermagem.pdf

9. Camillo SO, Maiorino FT, Chaves LC. O ensino de enfermagem sobre HIV/Aids sob a ótica da cidadania. Rev Gaúch Enferm [Internet]. 2013 [acesso em 2018 jul 11];34(3):117-23. Disponível em: http://www.scielo.br/pdf/rgenf/v34n3/a15v34n3.pdf

10. Val LF, Silva JAS, Rincón LA, Lima RHA, Barbosa RL, Nichiata LYI. Estudantes do ensino médio e o conhecimento em HIV/AIDS: que mudou em dez anos?. Rev Esc Enferm USP [Internet]. 2013 [acesso em 2018 jul 11];47(3):702-8. Disponível em: http://www.scielo.br/scielo.php?script=sci_arttext\&pid=S0080$62342013000300702 \& \operatorname{lng}=\mathrm{pt}$

11. Fowler G, Austoker J. Screening. In: Detels R, Holland WW, McEwen J, Omenn GS. Oxford textbook of public health. New York: Oxford University Press; 1997. p.1583-99.

12. Costa JSD, Victora CG. O que é "um problema de saúde pública"? Rev Bras Epidemiol [Internet]. 2006 [acesso em 2018 jul 11];9(1):144-6. Disponível em: http://www.scielo.br/scielo.php?script=sci_arttext\&pid=S1415-790X2006000100018\&lng=pt

13. Padilha MI, Bellaguarda MLR, Nelson S, Maia ARC, Costa R. O uso das fontes na condução da pesquisa histórica. Texto \& Contexto Enferm [Internet]. 2017 [acesso em 2018 jul 11];26(4):e2760017. Disponível em: http://dx.doi.org/10.1590/0104-07072017002760017

14. Prado ML, Gelbcke FL. Catálogo 1982-1997: trabalhos de conclusão: curso de graduação em enfermagem [monografia]. Florianópolis: Universidade Federal de Santa Catarina; 1999. 179 p.

15. Guedes HTV, Guedes JC. Avaliação, pelos estudantes, da atividade "trabalho de conclusão de curso" como integralização do eixo curricular de iniciação à pesquisa científica em um curso de medicina. Rev Bras Educ Méd [Internet]. 2012 [acesso em 2018 set 28];36(2):162-71. Disponível em: http://www.scielo.br/pdf/rbem/v36n2/03.pdf

16. Cunha C, Maciel TC. Assistência de enfermagem ao portador do HIV e doente de AIDS [monografia]. Florianópolis: Universidade Federal de Santa Catarina; 1990. 88 p. 
Produção acadêmica da enfermagem acerca dos temas hiv e aids: um estudo histórico-social | 16

17. Ministério da Saúde (BR), Departamento de Vigilância, Prevenção e Controle das IST, do HIV/Aids e das Hepatites Virais. História da Aids - 1982 [Internet]. 2018 [acesso em 2018 set 28]. Disponível em: http://www.aids.gov.br/pt-br/noticias/historia-da-aids-1982

18. Rodrigues-Júnior AL, Ruffino-Netto A, Castilho EA. Spatial distribution of the human development index, HIV infection and AIDS-Tuberculosis comorbidity: Brazil, 1982 - 2007. Rev Bras Epidemiol [Internet]. 2014 [acesso 2018 set 28];17(Suppl 2):204-15. Disponível em: http://www.scielo.br/scielo.php?script=sci_arttext\&pid=S1415-790X2014000600204\&lng=en\&nrm=iso

19. Governo de Santa Catarina, Secretaria da Saúde, Diretoria de Vigilância Epidemiológica (Dive). HIV entre adultos jovens cresce $43 \%$ em Santa Catarina nos últimos três anos, alerta Secretaria da Saúde [Internet]. Florianópolis; 2017 [acesso em 2018 set 28]. Disponível em: http://www.sc.gov.br/index.php/noticias/temas/saude/hiv-entre-adultos-jovens-cresce-43-em-santacatarina-nos-ultimos-tres-anos

20. Kirschner AR. Significando as formas de infeção e a relação com a gestão do cuidado a pessoa com HIV/Aids [dissertação]. Florianópolis: Universidade Federal de Santa Catarina; 2014. 154p.

21. Santos PHS, Reis LAD. Subnotificação de acidentes de trabalho em profissionais de enfermagem: revisão integrativa. Rev Enferm UFPE On Line [Internet]. 2016 [acesso em 2018 jul 11];10(2):640-6. Disponível em: https://periodicos.ufpe.br/revistas/revistaenfermagem/article/view/11000/12361

22. Machado JMH, Assunção AA, organizadores. Panorama da saúde dos trabalhadores da saúde. Belo Horizonte: UFMG; 2012. 164p.

23. Brasil. Ministério da Saúde. Gabinete do Ministro. Portaria n. 104, de 25 de janeiro de 2011. Dispõe sobre a definição das terminologias adotadas em legislação nacional, conforme o disposto no Regulamento Sanitário Internacional 2005 [Internet]. 2005 [acesso em 2018 jun 16]. Disponível em: http://bvsms.saude.gov.br/bvs/saudelegis/gm/2011/prt0104_25_01_2011.html

24. Donatelli S, Vilela RAG, Almeida IM, Lopes MGR. Acidente com material biológico: uma abordagem a partir da análise das atividades de trabalho. Saúde Soc [Internet]. 2015 [acesso em 2018 jul 11];24(4):1257-72. Disponível em: http://www.scielo.br/pdf/sausoc/v24n4/1984-0470-sausoc-24-0401257.pdf

25. Redmond AM, McNamara JF. The road to eliminate mother-to-child HIV transmission. J Pediatr [Internet]. 2015 [acesso em 2018 jun 16];91(6):509-11. Disponível em: http://www.scielo.br/pdf/jped/v91n6/0021-7557-jped-91-06-0509.pdf

26. Fonseca PL, Iriart JAB. Aconselhamento em DST/Aids às gestantes que realizaram o teste anti-HIV na admissão para o parto: os sentidos de uma prática. Interface Comun Saúde Educ [Internet]. 2012 jun [acesso em 2018 set 28];16(41):395-407. Disponível em: http://www.scielo.br/scielo.php?script=sci_arttext\&pid=S1414-32832012000200009\&lng=pt\&nrm=iso 
27. Cartaxo CMB, Nascimento CAD, Diniz CMM, Brasil DRPA, Silva IF. Gestantes portadoras de HIV/AIDS: aspectos psicológicos sobre a prevenção da transmissão vertical. Estud Psicol [Internet]. 2013 set [acesso em 2018 jul 11];18(3):419-27. Disponível em: http://www.scielo.br/scielo.php?script=sci_arttext\&pid=S1413-294X2013000300002\&lng=en\&nrm=iso

28. Amoras BCA, Campos AR, Beserra EP. Reflexões sobre vulnerabilidade dos adolescentes a infecções sexualmente transmissíveis. PRACS Rev Eletrônica Humanid Curso Ciênc Soc UNIFAP [Internet]. 2015 jan-jul [acesso em 2018 set 28];8(1):163-71. Disponível em: https://periodicos.unifap.br/index.php/pracs/article/view/1668

\section{Autor correspondente}

Stéfany Petry

E-mail: stefanypetry@hotmail.com

Endereço: Rua Aracuã, 34 - Pantanal, Florianópolis-SC, Brasil.

CEP: 88040310

\section{Contribuições de Autoria}

1 - Stéfany Petry

Concepção e planejamento da pesquisa; obtenção, análise e interpretação dos dados; redação e revisão crítica.

2 - Maria Itayra Padilha

Concepção e planejamento da pesquisa; análise e interpretação dos dados; redação e revisão crítica.

3 - Ana Rosete Maia

Contribuições: Redação e revisão crítica.

4 - Gabriela Beims Gapski

Análise e interpretação dos dados.

\section{Como citar este artigo}

Petry S, Padilha MI, Maia AR, Gapski GB. Produção acadêmica da enfermagem acerca dos temas hiv e aids: um estudo histórico-social. Rev. Enferm. UFSM. 2019 [Acesso em: 2019 jun 15];vol 9 ex:p1-p17. DOI:https://doi.org/10.5902/2179769235114 